\title{
Differential inflammasome activation predisposes to acute-on-chronic liver failure in human and experimental cirrhosis with and without previous decompensation
}

\author{
Sofia Monteiro, ${ }^{1,2}$ Josephine Grandt, ${ }^{3}$ Frank Erhard Uschner (D) , ${ }^{4}$ Nina Kimer (D) , ${ }^{3}$ \\ Jan Lysgård Madsen, ${ }^{5}$ Robert Schierwagen (1) , ${ }^{4}$ Sabine Klein, ${ }^{4}$ Christoph Welsch, \\ Liliana Schäfer, ${ }^{6}$ Christian Jansen, ${ }^{1}$ Joan Claria (1) ${ }^{7,8}{ }^{\text {José Alcaraz-Quiles, }}{ }^{9}$ \\ Vicente Arroyo, ${ }^{7}$ Richard Moreau, ${ }^{10}$ Javier Fernandez, ${ }^{7,8}$ Flemming Bendtsen, ${ }^{3}$ \\ Gautam Mehta (D) , ${ }^{11}$ Lise Lotte Gluud, ${ }^{3}$ Søren Møller, ${ }^{12}$ Michael Praktiknjo (D) , \\ Jonel Trebicka (iD 4,7
}

Additional material is published online only. To view please visit the journal online (http://dx.doi.org/10.1136/ gutjnl-2019-320170).

For numbered affiliations see end of article.

Correspondence to Dr Jonel Trebicka, Department of Internal Medicine 1, University of Frankfurt, Frankfurt am Main D 60590, Germany: Jonel.Trebicka@kgu.de

SM, JG and FEU are joint first authors.

Received 25 October 2019 Revised 18 March 2020 Accepted 21 March 2020 Published Online First 2 April 2020
Check for updates

(C) Author(s) (or their employer(s)) 2021. Re-use permitted under CC BY-NC. No commercial re-use. See rights and permissions. Published by BMJ.

To cite: Monteiro S, Grandt J Uschner FE, et al. Gut

2021:70:379-387.

\section{ABSTRACT}

Objective Systemic inflammation predisposes acutely decompensated (AD) cirrhosis to the development of acute-on-chronic liver failure (ACLF). Supportive treatment can improve $A D$ patients, becoming recompensated. Little is known about the outcome of patients recompensated after AD. We hypothesise that different inflammasome activation is involved in ACLF development in compensated and recompensated patients.

Design 249 patients with cirrhosis, divided into compensated and recompensated (previous AD), were followed prospectively for fatal ACLF development. Two external cohorts $(n=327)$ (recompensation, $A D$ and ACLF) were included. Inflammasome-driving interleukins (ILS), IL-1 $\alpha$ (caspase-4/11-dependent) and IL-1 $\beta$ (caspase-1dependent), were measured. In rats, bile duct ligationinduced cirrhosis and lipopolysaccharide exposition were used to induce $A D$ and subsequent recompensation.

$\mathrm{IL}-1 \alpha$ and IL- $1 \beta$ levels and upstream/downstream gene expression were measured.

Results Patients developing ACLF showed higher baseline levels of ILs. Recompensated patients and patients with detectable ILs had higher rates of ACLF development than compensated patients. Baseline CLIF-C (European Foundation for the study of chronic liver failure consortium) AD, albumin and IL- $1 \alpha$ were independent predictors of ACLF development in compensated and CLIF-C AD and IL- $1 \beta$ in recompensated patients. Compensated rats showed higher IL-1 $\alpha$ gene expression and recompensated rats higher IL-1 $\beta$ levels with higher hepatic gene expression. Higher IL-1 $\beta$ detection rates in recompensated patients developing ACLF and higher IL- $1 \alpha$ and IL-1 $\beta$ detection rates in patients with $A C L F$ were confirmed in the two external cohorts.

Conclusion Previous AD is an important risk factor for fatal ACLF development and possibly linked with inflammasome activation. Animal models confirmed the results showing a link between ACLF development and IL- $1 \alpha$ in compensated cirrhosis and IL- $1 \beta$ in recompensated cirrhosis.

\section{Significance of this study}

What is already known on this subject?

- Acute-on-chronic liver failure (ACLF) is a syndrome with high short-term mortality.

- Its development is associated with systemic inflammation.

What are the new findings?

- This study shows that interleukin (IL)$1 \alpha$ is an independent predictor of fatal ACLF development in compensated and IL-1 $\beta$ in recompensated experimental and human cirrhosis, suggesting that distinct inflammasome activation seems to influence fatal ACLF development.

How might it impact on clinical practice in the foreseeable future?

- These results contribute to the understanding of inflammasome activation in fatal ACLF and might pave the way to the development of therapeutic targets to prevent fatal ACLF.

\section{INTRODUCTION}

In cirrhosis, acute-on-chronic liver failure (ACLF) is a recently described syndrome characterised by acute decompensation (AD), organ failure and high short-term mortality. ${ }^{1-4}$ The pathways leading to this syndrome are not yet fully known. Systemic inflammation (SI) occurs in the setting attributable to translocation of proinflammatory signals from the intestinal lumen to systemic circulation and/or the release of damage-associated molecular patterns, ${ }^{5-7}$ with a burst of proinflammatory mediators. The direct deleterious effects of these proinflammatory mediators on organ microcirculation and cell physiology haemostasis can lead to organ failure. ${ }^{18}$ At any stage of cirrhosis, patients can develop $\mathrm{AD},{ }^{19}$ but patients only develop ACLF when SI is broadly activated. ${ }^{19}$ Under standard 
medical therapy of $\mathrm{AD}$, some patients can improve, becoming clinically stable and recompensated. Recent data suggest that the ACLF prevalence among outpatients is high. ${ }^{10}$ Additionally, almost half of the CANONIC cohort developed ACLF without a precipitating event, ${ }^{1}$ highlighting that either triggers are not completely known or in some cases SI can be so high that ACLF can develop without external triggers.

Compensated and recompensated (ie, with a history of decompensation) outpatients present in similar clinical conditions in real-life clinical practice. However, differences in risk of ACLF development between compensated and recompensated patients have not been studied yet. ${ }^{10}$ Although it seems consensual that SI is a hallmark of ACLF, the dynamics of SI after successfully treated $\mathrm{AD}$ (recompensation) has not been described and its role in ACLF development remains unclear.

Inflammasome activation is a hallmark of inflammatory response of the innate immune system leading to release of interleukin (IL)- $1 \alpha$ and IL-1 $\beta .^{11}$ Activation of inflammasomes via canonical pathway involves sensing of triggers, such as dangerassociated molecular patterns or pathogen-associated molecular patterns, cytosolic assembly of inflammasome, and consequently caspase-1-dependent cleaving of biologically inactive pro-IL-1 $\beta$ to its active form IL-1 $\beta$. Subsequently, systemic inflammatory response via IL- 6 and $C$ reactive protein gets set in motion. ${ }^{12} 13$ Of note, cytosolic lipopolysaccharide (LPS) is a known trigger for non-canonical inflammasome signalling involving caspase$11 / 4 / 5$, subsequently leading to pyroptosis and release of the alarm in IL-1 $\alpha$. Therefore, IL- $1 \alpha$ contributes to local inflammatory response. ${ }^{14}$

The aim of the present study was therefore to evaluate inflammasome activation, assessed by proinflammatory cytokines IL- $1 \alpha$ and IL- $1 \beta$, of compensated and recompensated patients and their role in fatal ACLF development. We hypothesise that SI is a prerequisite and indicative of the development of ACLF in states of compensated and recompensated patients.

\section{PATIENTS AND METHODS}

\section{Patients and data collection}

In this prospective, single-centre study, 249 patients with liver cirrhosis were electively referred for hepatic venous pressure gradient (HVPG) measurement to the Department of Clinical Physiology and Gastrounit, Hospital Hvidovre, Denmark, between 2002 and 2016, per protocol for study purposes. The inclusion criteria were age $>18$ years, and a diagnosis of cirrhosis based on clinical characteristics combined with HVPG and histology, biochemical, ultrasonographic or endoscopic findings. The exclusion criteria were age $<18$ years, $\mathrm{AD}$ at the time of inclusion, malignancy and acute bacterial infection. General clinical characteristics are displayed in online supplementary table 1 .

The patients were then divided into two groups according to their history of decompensation. ${ }^{15}$ Compensated patients were defined as never having a decompensating event. Recompensated patients were defined as decompensated patients (previous episode of ascites) that were clinically inapparent without ascites or with controlled ascites using medical treatment at time of inclusion.

The primary endpoint was fatal ACLF, defined as ACLF development leading to death.

Blood samples were collected at the time of the enrolment and patients were followed until October 2017. The ACLF definition according to the European Foundation for the study of chronic liver failure consortium (CLIF-C) was established in 2013, and the ACLF diagnosis was therefore established retrospectively. ${ }^{12}$ Records of non-fatal ACLF were not available. Biochemical blood analyses were performed using standard tests. Written informed consent was obtained from included patients.

A subcohort of 48 patients from a previous study were included as an external cohort (EC1) to confirm the results for the recompensation group. ${ }^{16}$ These patients were admitted and recompensated from $\mathrm{AD}$ at the Department of Internal Medicine I, University Hospital Bonn, Germany. Blood samples were used to measure circulating levels of IL-1 $\beta$. The patients were followed up for the development of ACLF.

Another external cohort (EC2) of 279 patients with AD liver cirrhosis from a prospective, multicentre study $^{1}$ were analysed for serum levels of IL- $1 \alpha$ and IL-1 $\beta$ and presence of ACLF to evaluate the inflammasome activation in ACLF. The patients from EC2 were not followed up.

There were no funds or time allocated for patient or public involvement, so we were unable to invite patients to comment on the study design, development of patient relevant outcomes or interpretation of the results. Patients were not invited to contribute to the writing or editing of this document for readability or accuracy.

\section{Assessment of circulating levels of ILs}

At the initial evaluation peripheral blood was collected from all the patients included in this study. After collection, blood samples were centrifuged at $4^{\circ} \mathrm{C}$ and serum samples were stored at $-80^{\circ} \mathrm{C}$. Serum concentrations of IL- $1 \alpha$ and IL- $1 \beta$ were assessed with DuoSet ELISA Kits (R\&D Systems, Minneapolis, USA) according to the manufacturer's instructions (online supplementary table 2) at the Department of Internal Medicine I in Bonn, Germany. The IL levels were quantified in undiluted serum samples; serum from two patients was used as control accessing variability within each ELISA plate.

The range of detection was $7.81-500 \mathrm{pg} / \mathrm{mL}$ for IL- $1 \alpha$ and $3.91-250 \mathrm{pg} / \mathrm{mL}$ for IL-1 3 . Undetectable levels were assigned a value equal to the lower limit of detection. ${ }^{1718}$ For values higher than the upper limit of detection, concentrations were calculated using GraphPad Prism V.5.00 (GraphPad Prism Software, La Jolla, USA).

In EC1, IL-1 $\beta$ was measured using the highly sensitive Luminex MAGPIX system (EMD Millipore, Darmstadt, Germany) with ProcartaPlex Mix\&Match Human 15-Plex (eBiosciences, Carlsbad, California) according to the manufacturer's instructions. ${ }^{16}$ In EC2, IL- $1 \alpha$ and IL-1 $\beta$ were assessed in a Luminex 100 system (Luminex, Austin, Texas) using a custom-made MILLIPLEX MAP Human Cytokine/Chemokine Magnetic Bead Panel (Merck Millipore, Billerica, Massachusetts). Briefly, $25 \mu \mathrm{L}$ of diluent and $25 \mu \mathrm{L}$ of plasma or leucocyte supernatant were added to each well before the addition of premixed microbeads $(25 \mu \mathrm{L})$. The plate was incubated overnight at $4^{\circ} \mathrm{C}$ with shaking, then washed and reincubated with $25 \mu \mathrm{L}$ of detection antibody for 1 hour. The plate was washed again and incubated with $25 \mu \mathrm{L}$ of streptavidin-phycoerythrin for $30 \mathrm{~min}$. The plate was finally washed twice and the beads were resuspended with $100 \mu \mathrm{L}$ of sheath fluid and analysed in the Luminex 100 system. Readouts were detected as mean fluorescence intensity by the instrument, and values were subsequently converted to $\mathrm{pg} / \mathrm{mL}$ by extrapolation from a set of standards that were run simultaneously in the assay. The intra-assay and interassay coefficients of variation ranged from 1.6 to 3.3 and from 6.7 to 16.8 , respectively. ${ }^{19}$ 


\section{Animal experiments}

We used male wild-type (WT) Sprague Dawley rats in our experiments, according to the guidelines and regulations approved by LANUV, the committee responsible for animal studies in North Rhine-Westphalia. All animals received water and chow ad libitum. Sprague Dawley rats were housed in a controlled environment (standard IVC cage, 12-hour light/dark, temperature $22^{\circ} \mathrm{C}-24^{\circ} \mathrm{C}$ ) and fed standard rat chow (ssniff, Soest, Germany). The experiments were performed during the light cycle.

\section{Cholestatic model of fibrosis}

Bile duct ligation (BDL) was performed in WT rats with an initial body weight (BW) of 180-200 g. ${ }^{20} 21$ The AD model rats received a single intraperitoneal dose of LPS from Klebsiella pneumoniae (Sigma L4268, St Louis, USA), $0.03 \mathrm{mg} / \mathrm{kg}$ BW, 28 days after BDL. The rats were then sacrificed 0, 4, 24 and 72 hours after injection of LPS. Experiments of the AD model were performed at the Institute for Liver and Digestive Health, University College London, UK.

For the recompensation model, experiments were carried out 3 weeks after BDL. The recompensation group received a single intravenous dose of LPS from Escherichia coli O111:B4 (SigmaAldrich, St Louis, USA), $6.25 \mathrm{mg} / \mathrm{kg} \mathrm{BW}$ to induce AD. Rats then recompensated and were sacrificed at 4 weeks (day 28).

The rationale for the LPS dose design is described in online supplementary material 1 .

The compensation group did not receive LPS and consisted of rats without ascites at sacrifice after 4 weeks (day 28). Liver and serum samples were snap-frozen and stored at $-80^{\circ} \mathrm{C}$. Experiments of the compensation and recompensation model were performed at the Department of Internal Medicine I, University Hospital Bonn, Germany.

\section{Peripheral blood mononuclear cells}

Peripheral blood mononuclear cells (PBMCs) were isolated from the EDTA blood of compensated and recompensated rats using Pancoll gradient centrifugation (PAN-Biotech, Aidenbach, Germany) as previously described. ${ }^{22}$ Cells were suspended in Roswell Park Memorial Institute (RPMI) 16/40 medium $+10 \%$ fetal calve serum + dimethyl sulfoxide (Gibco, Life Technologies, Carlsbad, USA) and stored at $-80^{\circ} \mathrm{C}$.

\section{Assessment of circulating IL levels in rodents}

For the AD animal model, IL- $1 \alpha$ levels were assessed using Abcam kit (ab113350; Abcam Cambridge, UK) (array sensitivity $<15 \mathrm{pg} / \mathrm{mL}$ ) according to the manufacturer's instructions.

Circulating IL- $1 \alpha$ and IL- $1 \beta$ levels in rodents of the compensation and recompensation models were measured in $25 \mu \mathrm{L}$ of plasma using a multiplexed bead-based immunoassay (MILLIPLEX MAP Cytokine/Chemokine Magnetic Bead Panel; Merck Millipore, Darmstadt, Germany) on a Luminex 100 Bioanalyzer (Luminex, Austin, Texas) as previously described. ${ }^{23}$ The readouts were analysed with MILLIPLEX Analyst software (Merck Millipore), and a five-parameter logistic regression model was used to calculate the concentration of each sample $(\mathrm{pg} / \mathrm{mL})$.

\section{CRNA microarray}

RNA isolation from rat liver and PBMC samples was performed as previously described. ${ }^{21}$ Complementary RNA (cRNA) microarray was done by OakLabs (Genome-wide Gene Expression Services, Hennigsdorf, Germany) using OakLab's Microarray XS Agilent microarray (Agilent Technologies, Santa Clara, USA). Summarised, Low Input Quick Amp Labeling
Kit (Agilent) was used to create fluorescent cRNA, and Gene Expression Hybridization Kit (Agilent) was used for hybridisation on the microarrays. Fluorescence signals were detected using SureScan Microarray Scanner (Agilent). Statistical parameters were computed between groups, and the results are shown as fold change and visualised by heat maps. P values were calculated using paired t-test and corrected according to the adaptive Benjamini-Hochberg procedure. False discovery rate was computed and indicated as q-values. Only genes with low q-value $(<0.05)$ were used for further analyses.

\section{Messenger RNA expression analysis}

Total RNA from the AD model was extracted from snapfrozen mouse liver and cell samples using standard techniques. Subsequently, gene expression was analysed according to the manufacturer's protocols using TaqMan probes for IL-1 $\alpha$ (Rn.PT.58.35304224) and Rplp0 (housekeeper-Rn. PT.39a.22214840) (Integrated DNA Technologies, Coralville, Iowa, USA).

\section{Statistical analysis}

Data are presented as median and ranges or absolute frequency and percentage, if not otherwise specified. Mann-Whitney and Kruskal-Wallis tests were used for unpaired comparisons. Receiver operating characteristic analysis was used to calculate cut-off values. Kaplan-Meier curves were used to analyse rates of ACLF development and compared using log-rank test. Univariate Cox regression was used to identify predictors of fatal ACLF. Significant predictors in univariate Cox regression were evaluated in multivariate Cox regression to identify the independent predictors. To avoid multicollinearity in multivariate Cox regression, the Model for End-Stage Liver Disease (MELD) and CLIF-C AD scores and their included parameters were not included simultaneously.

Unlike previous studies, we chose to include all patients into our analyses, regardless of the baseline IL concentration. To assess ILs as clinically feasible biomarkers for fatal ACLF, we therefore chose to stratify patients into those with detectable and undetectable levels of ILs.

The significance level for all tests was set at $\mathrm{p}<0.05$. Statistical analyses were performed using SPSS V.25.

\section{RESULTS}

\section{General characteristics of patients}

We included 249 patients $(73 \%$ male, 74\% with alcoholic cirrhosis, median age 60 years; online supplementary table 1). The median Child-Pugh, MELD and CLIF-C AD scores were only 7 (5-12), 10 (6-25) and 49 (33-67) points. At inclusion, none of the patients with alcoholic cirrhosis had acute alcoholic liver injury according to the National Institute on Alcohol Abuse and Alcoholism (NIAAA) criteria (at least three of the following: active alcoholism of 6 months $>40 \mathrm{~g} /$ day for women or $>60 \mathrm{~g} /$ day for men, bilirubin $>3 \mathrm{mg} / \mathrm{dL}$, aspartate aminotransferases (AST) $>50$ intentional units (IU), AST/alanine transaminase $($ ALT) $>1.4$, AST or ALT $>400$ IE). Patients with viral hepatitis were untreated at inclusion.

IL-1 $\alpha$ and IL-1 $\beta$ were detectable in $62(25 \%)$ and $78(31 \%)$ patients (table 1$)$. In patients with detectable levels, the median concentration of IL- $1 \alpha$ was $45.6 \mathrm{pg} / \mathrm{mL}$ (9.5-1573.8) and $89.2 \mathrm{pg} / \mathrm{mL}$ (8.5-768.2). In those with detectable IL-1 $\beta$, the numbers were $73.8 \mathrm{pg} / \mathrm{mL}(4.1-622.9)$ and $70.4 \mathrm{pg} / \mathrm{mL}(4.3-$ 1180.5 ) (table 1). There was no significant difference in the IL levels between the time eras (2002-2011 vs 2012-2016) 
Table 1 Characteristics of all patients stratified by compensated and recompensated cirrhosis

\begin{tabular}{|c|c|c|}
\hline & Compensated & Recompensated \\
\hline Parameter & $n=128$ & $n=121$ \\
\hline \multicolumn{3}{|l|}{ General condition } \\
\hline Age (years) & $61(31-82)$ & $60(35-77)$ \\
\hline Sex (male/female) & $92(72) / 36(28)$ & $91(75) / 30(25)$ \\
\hline $\begin{array}{l}\text { Aetiology of cirrhosis } \\
\text { (alcohol/viral/other) }\end{array}$ & $86(67) / 21(16) / 21(16)$ & $99(82) / 5(4) / 17(14)$ \\
\hline BMI $\left(\mathrm{kg} / \mathrm{m}^{2}\right)$ & $25(13-45)$ & $24(16-41)$ \\
\hline \multicolumn{3}{|l|}{ Baseline scores } \\
\hline MELD + & $9(6-25)$ & $12(6-21)^{* * *}$ \\
\hline Child-Pugh (A/B/C) & $51(40) / 44(34) / 33(26)$ & $46(38) / 26(21) / 49(41)$ \\
\hline Child-Pugh & $6(5-12)$ & $7(5-12)$ \\
\hline CLIF-C AD§ & 47 (34-64) & $52(33-67)^{* * *}$ \\
\hline \multicolumn{3}{|l|}{ Baseline laboratory } \\
\hline $\mathrm{Hb}(\mathrm{gL})$ & $131(82-171)$ & $116(68-169)^{* * *}$ \\
\hline WCC $(\times 109 / L) \S$ & $6.7(2.4-17.2)$ & $7.3(1.8-23.3)$ \\
\hline Platelets (G/L)‡ & $147(29-453)$ & $152(36-647)$ \\
\hline Sodium (mmol/L) & 139 (118-146) & $136(114-145)^{* * *}$ \\
\hline Creatinine (mg/dL) & $0.8(0.4-2.2)$ & $0.9(0.4-2.7)^{* *}$ \\
\hline Bilirubin $(\mathrm{mg} / \mathrm{dL}) \dagger$ & $0.7(0.1-12.7)$ & $1.2(0.2-6.4)^{* * *}$ \\
\hline ALT $(\mathrm{U} / \mathrm{L}) \dagger$ & $30(7-311)$ & $30(10-136)$ \\
\hline Albumin $(g / L) \dagger$ & $35(15-47)$ & $28(18-43)^{* * *}$ \\
\hline INRT & $1.2(0.9-2.6)$ & $1.4(1.0-2.5)^{* * *}$ \\
\hline HVPG & $12(1.5-28)$ & $18(0.5-33)^{* * *}$ \\
\hline \multicolumn{3}{|l|}{ Baseline clinical } \\
\hline Ascites (no/yes) & $128(100) / 0(0)$ & $0(0) / 121(100)^{* * *}$ \\
\hline $\begin{array}{l}\text { Oesophageal varices (no - } \\
\text { grade I/grade II-III) }\end{array}$ & $110(86) / 19(14)$ & $62(51) / 59(49)^{*}$ \\
\hline \multicolumn{3}{|l|}{ Outcome } \\
\hline Mortality 1 year/overall & $9(7) / 29(23)$ & $30(25)^{*} / 62(51)^{* * *}$ \\
\hline $\begin{array}{l}\text { Fatal ACLF development } \\
1 \text { year/overall }\end{array}$ & $4(3) / 14(11)$ & $17(14) / 38(31)^{*}$ \\
\hline $\begin{array}{l}\text { Time to fatal ACLF } \\
\text { development (months) }\end{array}$ & $4(0-8)$ & $2(0-15)$ \\
\hline Follow-up time (years) & $2(0-6)$ & $1(0-11)^{* * *}$ \\
\hline \multicolumn{3}{|l|}{ Biomarkers } \\
\hline \multicolumn{3}{|l|}{ IL-1 $\alpha$} \\
\hline (Undetectable/detectable) & $97(76) / 31(24)$ & $90(74) / 31(26)$ \\
\hline Detectable (pg/mL) & $45.6(9.5-1573.8)$ & $89.2(8.5-768.2)$ \\
\hline \multicolumn{3}{|l|}{ IL-1 $\beta$} \\
\hline (Undetectable/detectable) & $95(74) / 33(26)$ & $77(63) / 45(37)$ \\
\hline Detectable $(\mathrm{pg} / \mathrm{mL})$ & $73.8(4.1-622.9)$ & $70.4(4.3-1180.5)$ \\
\hline
\end{tabular}

Data expressed as median (range) or absolute frequency (percentage).

${ }^{* * *} \mathrm{P}<0.001,{ }^{* *} \mathrm{P}<0.01,{ }^{*} \mathrm{P}<0.05$.

tData available in 246 patients.

¥Data available in 245 patients.

$\S$ Data available in 231 patients.

$A C L F$, acute-on-chronic liver failure; $A D$, acute decompensation; $A L T$, alanine transaminase; BMI, body mass index; CLIF-C, European Foundation for the study of chronic liver failure consortium; $\mathrm{Hb}$, haemoglobin; $\mathrm{HVPG}$, hepatic venous pressure gradient; IL, interleukin; INR, international normalised ratio; MELD, Model for EndStage Liver Disease; WCC, white cell count.

stratified for MELD ( $\leq 11$ vs $>11$ ), suggesting stability of the measured cytokines in the blood samples stored over the years of the study (online supplementary table 3 ).

In total, $88(35 \%)$ patients died. The most common cause of death was ACLF for 52 (58.5\%) patients. Other more common causes of death were malignancies (11.2\%) and cardiovascular events $(14.6 \%)$ (online supplementary table 4$)$. The trigger of
Table 2 Multivariate Cox regression analysis for fatal ACLF development in compensated patients

\begin{tabular}{|c|c|c|c|c|c|c|}
\hline \multirow[b]{2}{*}{ Parameter } & \multicolumn{3}{|c|}{ Univariate Cox regression } & \multicolumn{3}{|c|}{ Multivariate Cox regression } \\
\hline & $P$ value & HR & $95 \% \mathrm{Cl}$ & $P$ value & HR & $95 \% \mathrm{Cl}$ \\
\hline \multicolumn{7}{|l|}{$\begin{array}{l}\text { Compensated } \\
\text { cirrhosis }\end{array}$} \\
\hline CLIF-C AD & 0.002 & 1.175 & 1.061 to 1.301 & 0.052 & 1.106 & 0.999 to 1.224 \\
\hline $\mathrm{Hb}$ & 0.008 & 0.692 & 0.527 to 0.908 & & & \\
\hline Albumin & $<0.001$ & 0.220 & 0.095 to 0.512 & 0.010 & 0.872 & 0.786 to 0.967 \\
\hline Bilirubin & $<0.001$ & 1.454 & 1.193 to 1.772 & & & \\
\hline $\begin{array}{l}\mathrm{IL}-1 \alpha \\
\times 10^{-2}\end{array}$ & 0.011 & 1.257 & 1.055 to 1.499 & 0.013 & 1.248 & 1.048 to 1.486 \\
\hline $\begin{array}{l}\mathrm{IL}-1 \beta \\
\times 10^{-2}\end{array}$ & 0.253 & & & & & \\
\hline
\end{tabular}

ACLF, acute-on-chronic liver failure; $A D$, acute decompensation; CLIF-C, European Foundation for the study of chronic liver failure consortium; Hb, haemoglobin; IL, interleukin.

ACLF is unknown in $63.5 \%$ of cases. The most common of known triggers of fatal ACLF was infection in $68.4 \%$ of patients (25\% including all cases) (online supplementary table 5). The median time to fatal ACLF was 17 months (0-137).

\section{Characteristics of compensated and recompensated patients}

Patients were divided into compensated (no previous AD) and recompensated (previous ascites). Fifty-one per cent had compensated $(n=128)$ and $49 \%$ had recompensated $(n=121)$ cirrhosis.

Compared with compensated patients, the recompensated patients had significantly higher median MELD and CLIF-C AD (including differences in their components), lower haemoglobin, lower albumin, and higher alkaline phosphatase (table 1). In addition, the proportion of patients with large oesophageal varices was higher in recompensated $(49 \%, n=59)$ than compensated $(14 \%, \mathrm{n}=19)$ cirrhosis.

Recompensated patients had a significantly higher rate of fatal ACLF $(31 \%, n=38$ vs $11 \%, n=14)$ and overall mortality $(51 \%$, $\mathrm{n}=62$ vs $23 \%, \mathrm{n}=29)$ compared with compensated patients (table 1). The proportion of patients with detectable IL- $1 \alpha$ was 24\% among patients with compensated cirrhosis and $26 \%$ among patients with recompensated cirrhosis. The proportion with detectable IL-1 $\beta$ in the two groups was $26 \%$ and $37 \%$ (table 1).

\section{Predictors of fatal ACLF development}

Based on univariable and multivariable Cox regression analyses (tables 2 and 3), the baseline CLIF-C AD (HR 1.106, $\mathrm{p}=0.052)$, albumin (HR 0.872, p=0.010) and IL-1 $\alpha(\mathrm{HR} 1.248, \mathrm{p}=0.013)$ were independent predictors of fatal ACLF in the compensated cohort. In recompensated cirrhosis, IL-1 $\beta$ (HR 1.184, $p=0.011)$ and CLIF-C AD (HR 1.079, p=0.006) were the only independent predictors of fatal ACLF.

\section{Association of serum levels of IL and fatal ACLF development in compensated and recompensated patients}

Recompensated patients showed a significantly higher rate of fatal ACLF compared with compensated patients (figure 1A). Patients developing fatal ACLF showed significantly higher baseline detection rate of proinflammatory IL (online supplementary table 6).

Kaplan-Meier curves showed a higher rate of fatal ACLF in patients with detectable IL- $1 \alpha$ and IL- $1 \beta$ among recompensated 
Table 3 Multivariate Cox regression analysis for fatal ACLF development in recompensated patients

\begin{tabular}{|c|c|c|c|c|c|c|}
\hline \multirow[b]{2}{*}{ Parameter } & \multicolumn{3}{|c|}{ Univariate Cox regression } & \multicolumn{3}{|c|}{ Multivariate Cox regression } \\
\hline & $P$ value & HR & $95 \% \mathrm{Cl}$ & $P$ value & HR & $95 \% \mathrm{Cl}$ \\
\hline \multicolumn{7}{|c|}{ Recompensated cirrhosis } \\
\hline CLIF-C AD & 0.010 & 1.072 & 1.017 to 1.129 & 0.006 & 1.079 & 1.022 to 1.139 \\
\hline $\mathrm{Hb}$ & 0.738 & & & & & \\
\hline Albumin & 0.026 & 0.452 & 0.224 to 0.910 & & & \\
\hline Bilirubin & 0.776 & & & & & \\
\hline $\mathrm{IL}-1 \alpha \times 10^{-2}$ & 0.007 & 1.270 & 1.069 to 1.510 & & & \\
\hline IL-1 $\beta \times 10^{-2}$ & 0.007 & 1.183 & 1.047 to 1.337 & 0.011 & 1.184 & 1.039 to 1.348 \\
\hline
\end{tabular}

ACLF, acute-on-chronic liver failure; $A D$, acute decompensation; CLIF-C, European Foundation for the study of chronic liver failure consortium; $\mathrm{Hb}$, haemoglobin.

patients (online supplementary figure $1 \mathrm{~A}, \mathrm{~B}$ ). Likewise, patients with a high CLIF-C AD score ( $\geq 50$ points) had higher rates of fatal ACLF (figure 1B).

In compensated patients fatal ACLF development was similar between patients with detectable and undetectable IL- $1 \alpha$ and IL-1 $\beta$ (online supplementary figure $1 \mathrm{~A}$ and $\mathrm{B}$ ). To stratify patients at highest risk in the compensated group, we therefore combined detectable IL- $1 \alpha$ with CLIF-C AD score. The data show that patients with detectable levels of IL- $1 \alpha$ and CLIF-C $\mathrm{AD} \geq 50$ had significantly higher mortality (figure $1 \mathrm{C}$ ).

Similarly, we stratified the recompensated patients for IL-1 $\beta$ and CLIF-C AD score. Kaplan-Meier analysis shows clearly that patients with undetectable IL-1 $\beta$ levels and CLIF-C AD $<50$ had the best outcome (figure 1D).

Area under the receiver operating characteristics (AUROC) analysis for fatal ACLF development showed higher area under the curve (AUC) for Child-Pugh score, MELD, MELD-Na and CLIF-C AD score compared with IL- $1 \alpha$ and IL- $1 \beta$ alone. In compensated patients a combination of CLIF-C AD and IL- $1 \alpha$ and for recompensated patients a combination of CLIF-C AD and IL-1 $\beta$ showed slightly higher AUC compared with CLIF-C $\mathrm{AD}$ alone and therefore the highest AUC, respectively (online supplementary figures 2 and 3 ).
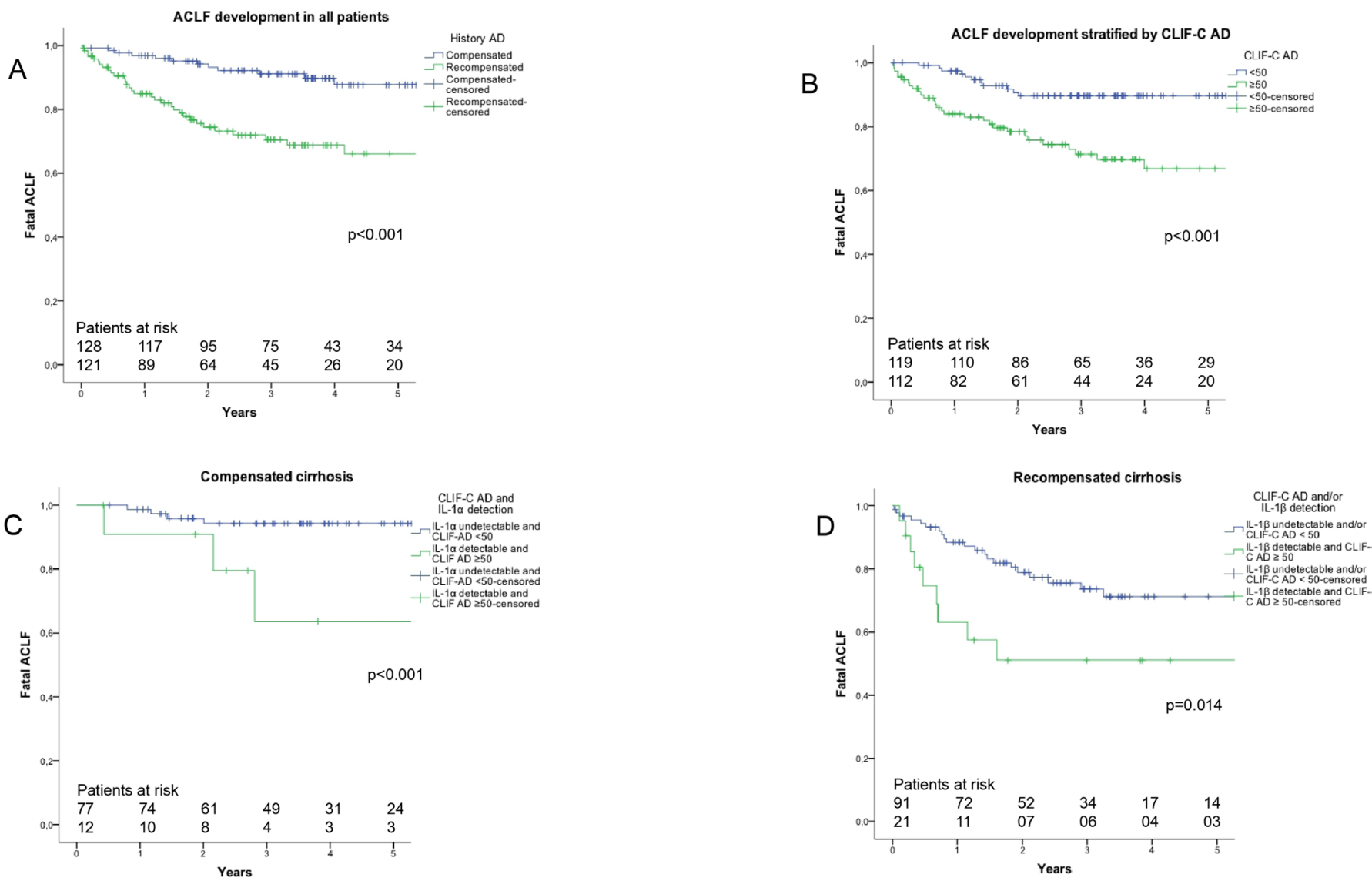

Figure 1 Kaplan-Meier curve for fatal ACLF development stratified into (A) compensated vs recompensated patients; (B) patients with CLIF-C AD score $<50$ vs $\geq 50$; (C) compensated patients with undetectable levels of IL- $1 \alpha$ and CLIF-C AD score $<50$ vs compensated patients with detectable levels of IL- $1 \alpha$ and CLIF-C AD score $\geq 50$; and (D) recompensated patients with undetectable levels of IL- $1 \beta$ and/or CLIF-C AD score $<50$ vs recompensated patients with detectable levels of IL-1 $\beta$ and CLIF-C AD score $\geq 50$. ACLF, acute-on-chronic liver failure; $A D$, acutely decompensated; CLIF-C, European Foundation for the study of chronic liver failure consortium; IL, interleukin. 


\section{External cohorts}

\section{External cohort 1}

In total, 48 patients recompensated from $\mathrm{AD}$ were included. The median age was 59 (18-80) years, with 27 (56\%) male patients. The main aetiology of cirrhosis was alcoholic (56\%). The median MELD was 9 (7-26), with most patients (75\%) classified as Child-Pugh stage B. During follow-up, 16 (33\%) patients developed ACLF. Patients developing ACLF showed significantly higher CLIF-C AD score (50 vs 45, p<0.05) compared with those without ACLF development. Other general characteristics were not significantly different. Importantly, using the highly sensitive assay, IL-1 $\beta$ levels were significantly higher and more frequently detectable in patients with ACLF development compared with those without ACLF development (69\% vs 34\%, $\mathrm{p}<0.05$ ) (online supplementary table 7 ). Vice versa, patients with detectable IL-1 $\beta$ detection showed significantly higher rates of ACLF development, confirming the role of IL-1 $\beta$ in recompensated patients (online supplementary figure 4).

\section{External cohort 2}

In total, $279 \mathrm{AD}$ patients (172 (61\%) male, mean age 58 years) were included. The main aetiologies were alcoholic $(n=140$, $50 \%)$ and chronic viral $(\mathrm{n}=82,29 \%)$ cirrhosis. In total, 178 (64\%) patients had ACLF at admission. Patients with ACLF showed higher mean Child-Pugh $(9 \pm 7$ vs $11 \pm 2)$ and MELD ( $16 \pm 6$ vs $27 \pm 7)$ compared with patients without ACLF. Importantly, patients with ACLF showed significantly higher rates of detectable IL- $1 \alpha$ (60\% vs 33\%) and IL-1 $(16 \%$ vs $8 \%$ ) compared with patients without ACLF. However, the detection rates of IL-1 $\beta$ are lower compared with the study cohort, which is likely attributable to using a different, less sensitive assay (online supplementary table 8).

\section{IL-1 $\alpha$ expression in early AD of experimental cirrhosis}

In the animal model of $\mathrm{AD}$ (figure $2 \mathrm{~A}$ ), we identified an early response in the hepatic IL- $1 \alpha$ gene expression with subsequent systemic IL- $1 \alpha$ release 4 hours after stimulation with LPS. The increased expression was lowered after 24 and 72 hours (figure 2B,C).

\section{IL-1 $\alpha$ and IL-1 $\beta$ expression in the recompensated experimental model}

When assessing the gene expression of IL-1 $\alpha$, we found no difference between the compensated and recompensated models in the liver, but the IL- $1 \alpha$ gene expression was significantly reduced in PBMC from recompensated compared with compensated animals (figure 2D). Serum levels were not significantly different between the two groups, suggesting IL- $1 \alpha$ as an early response in $\mathrm{AD}$ (figure $2 \mathrm{E}$ ).

Gene expression of IL-1 $\beta$ in liver tissue was significantly increased in recompensated compared with compensated rats (figure $2 \mathrm{~F}$ ). There was no significant difference in the gene expression in PBMC between the two groups (figure 2F). Serum levels of IL-1 $\beta$ were significantly higher in recompensated compared with compensated rats, suggesting different IL-1 $\beta$ driven activation pathways once AD occurred (figure $2 \mathrm{G}$ ).

Detailed heat maps of expression of upstream and downstream signalling of IL- $1 \alpha$ and IL- $1 \beta$ are shown in figure $2 \mathrm{H}$. In PBMC of compensated rats, there was a marked downregulation of IL-1 $\beta$ downstream signalling (IL1r1, Irak4, Traf6, Ikbkb, Ccl4, Vcam1). Upstream signalling of IL- $1 \alpha$ and IL- $1 \beta$ was reduced as well. In the liver tissue, IL-1 $\beta$ upstream (Casp1) and downstream

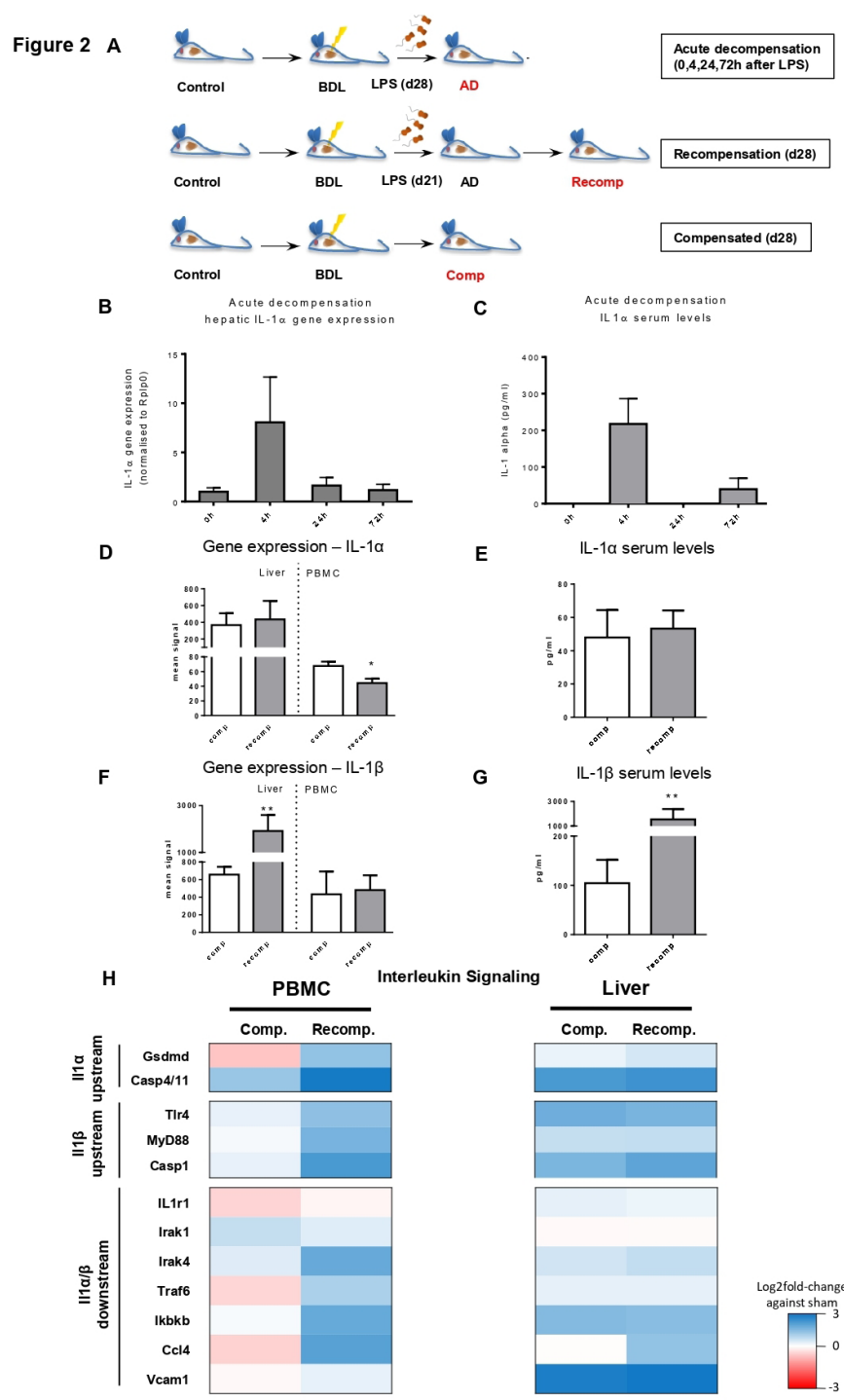

Figure 2 (A) Schematic of animal models for acute decompensation, recompensated and compensated cirrhosis. (B) Hepatic gene expression of IL-1 $\alpha$ in the acute decompensation model. (C) Circulating levels of IL-1 $\alpha$ in the acute decompensation model. (D) Gene expression of IL-1 $\alpha$ in the liver and PBMC in compensation and recompensation models. (E) Circulating levels of IL- $1 \alpha$ in the compensation and recompensation models. (F) Gene expression of IL-1 $\beta$ in the liver and PBMC in compensation and recompensation models. (G) Circulating levels of IL-1 $\beta$ in the compensation and recompensation models. (H) Heat map of gene expression of upstream and downstream signalling of IL-1 $\alpha$ and IL-1 $\beta$ in PBMC and liver in compensation and recompensation models. ${ }^{*} \mathrm{P}<0.05$ vs comp, ${ }^{*} \mathrm{P}<0.005$ vs comp. $A D$, acutely decompensated; $\mathrm{BDL}$, bile duct ligation; Comp, compensated; $\mathrm{d} 21$, day 21; d28, day 28; IL, interleukin; LPS, lipopolysaccharide; PBMC, peripheral blood mononuclear cell; Recomp, recompensated.

(Irak4, Ccl4, Vcam1) signalling were more expressed in recompensated than in compensated rats (figure $2 \mathrm{H}$ ).

\section{DISCUSSION}

This is the first study describing the differential mechanisms of SI predicting and probably inducing fatal ACLF in clinically stable patients with cirrhosis. Marked differences were identified between compensated and recompensated patients with cirrhosis. In the CANONIC and other cohorts, IL-6 and IL-8 
were strongly related with the frequency and severity of ACLF in $\mathrm{AD} .{ }^{17}$ This study adds to previous evidence and shows that in compensated patients IL-1 $\alpha$ and in recompensated patients IL-1 $\beta$ were independent predictors of fatal ACLF development. Importantly, the experimental animal models support these findings.

Although IL- $1 \alpha$ and IL- $1 \beta$ belong to the same family of inflammasome-driving ILs that act on the same receptor (IL1R1), they play different roles in SI. ${ }^{24-26}$ IL-1 $\alpha$ has constitutive and induced expression in a wide variety of cell types (not only in haematopoietic cells) and locations (cytoplasm, nucleus and the plasma membrane). ${ }^{27-29}$ Furthermore, pro-IL-1 $\alpha$, in contrast to pro-IL-1 $\beta$, is already fully biologically active. ${ }^{29}{ }^{30}$ Because of these properties IL-1 $\alpha$ initiates any inflammation. ${ }^{24} 253132$ Importantly, it is also released in inflammasome activation via the non-canonical pathway. ${ }^{33}$ This pathway involves LPS-induced activation of caspase-4/11 and activation of gasdermin, resulting in pyropoptosis. ${ }^{34}$ By contrast, IL- $1 \beta$ was identified as an independent risk factor for fatal ACLF in recompensated patients but not in compensated patients, highlighting another interesting facet of cirrhotic SI. The hypothesis of cirrhosis as a result of a continuous and progressive inflammatory state with increasing inflammatory activation with the progression of the disease is consistent with recent findings. We observed different inflammatory phenotypes in the stable compensated versus recompensated patients. However, those patients with $\mathrm{AD}$ who developed ACLF exhibited the highest number of abnormal markers of SI, indicating 'full-blown' inflammatory state with inflammasome activation. ${ }^{1835}$

Our study suggests that compensated and recompensated patients have different pathways of inflammasome activation and SI. We hypothesised that patients who primarily exhibit a high degree of immune incompetence and develop $\mathrm{AD}$ suffer an increased risk of ACLF development even if they are clinically recompensated. This hypothesis is supported by the higher rate of detectable IL-1 $\beta$ among recompensated patients, and further emphasised by our experimental results that clearly demonstrate increased levels of circulating levels of IL-1 $\beta$ in recompensated animals, signifying a high state of SI. The increased IL-1 gene expression specifically in liver tissue suggests hepatic inflammation to be the main origin of this elevated inflammatory state after recompensation.

The first external cohort (EC1) confirms and underlines the results of IL-1 $\beta$ in recompensation, as analysed in the study cohort. In these patients, recompensated from $\mathrm{AD}$, detectable levels of IL-1 $\beta$ were strongly associated with the development of ACLF. Moreover, EC1 complements the results of the study cohort by demonstrating the association of IL- $1 \beta$ and therefore canonical inflammasome activation with all (fatal and non-fatal) ACLF in recompensated patients.

The second external cohort (EC2) represents patients that are more advanced in the natural progression of liver disease presenting with ACLF. These patients presenting with ACLF display double the detection rate of both IL- $1 \alpha$ and IL- $1 \beta$, which is consistent with previous studies reporting a full-blown proinflammatory cytokine release in ACLF. ${ }^{17}{ }^{23}$ The differences in the detection rates of IL- $1 \beta$ between EC 2 and the study cohort are attributed to the use of different, less sensitive detection assay at a different laboratory, since in 'full blown' cytokine release in ACLF highly sensitive measurements are not thought to be necessary. However, the results of all three cohorts (study cohort, EC1, EC2) with more than 570 patients highlight the pathophysiology and the involvement of distinct inflammasome activation in different stages of liver cirrhosis independent of the assay used. Importantly, this study is not meant to describe the biomarkers of the disease, but rather to elaborate on the pathophysiological mechanisms leading to worse prognosis.

Overall, our findings were not only underlined by an external cohort but also nicely paralleled and confirmed in animal models, by analysing the first $\mathrm{AD}$ in an animal model and then showing that in recompensated and compensated animals the pathways of IL1 signalling are similarly activated as found in patients. This confirmation in the experimental data gives us confidence that there is a causal effect on the differential activation of the inflammasome system in cirrhosis according to the clinical situation. We expect that these results may be helpful in the guidance for new preventive initiatives and in the search for therapeutic targets for ACLF.

It has been shown that the inflammasome, especially nucleotide-binding domain leucine-rich repeat containing protein 3 (NLRP3), requires priming in addition to activation for cleavage and release of IL-1 $13 .^{3436}$ This might be an effect of prior AD in our patients and induced by stimulation with LPS in our animal models. This is supported by the increased expression of IL-1 receptor 1 (IL1R1) and tumour necrosis factor receptor-associated factor 6 (TRAF6) in our recompensation model compared with the compensation model. TRAF6 has been shown to mediate IL-1R signalling-induced non-transcriptional priming of the NLRP3 inflammasome. ${ }^{37}$ Recently, it has been shown that circulating levels of cell death markers (cK18, K18) were significantly lower in patients with cirrhosis with previous AD. This may reflect a dominant cell death (IL-1 $\alpha$ ) response in individuals without prior AD, but an NLRP3 response (IL-1 $\beta$ ) if there has been prior $\mathrm{AD} .^{38}$

Another possible pathophysiological mechanism could be the alternate pathway of inflammasome activation, which is unlike either the canonical or non-canonical pathways. It is initiated on stimulation with LPS independent of other activators of inflammasome. ${ }^{39}{ }^{40}$ However, these hypotheses require further studies.

As expected, MELD and CLIF-C AD were associated with the development of fatal ACLF. ${ }^{241}{ }^{42}$ However, the crucial role of IL- $1 \alpha$ and IL- $1 \beta$ is further underlined by their predictive value independent of MELD and CLIF-C AD scores. Assessing the cytokine profiles of compensated and recompensated patients with cirrhosis might help identify patients at risk for developing fatal ACLF beyond MELD and CLIF-C AD.

Our study describes a well-characterised and unique cohort, but has several limitations. First, we did not have access to data on alcohol abstinence and antiviral treatment in the follow-up period and to episodes of $\mathrm{AD}$, and therefore we cannot study the role of IL- $1 \alpha$ and IL- $1 \beta$ on transition from compensated to decompensated stages. In this study, fatal ACLF was diagnosed retrospectively, because ACLF was not defined yet at the time of the study initiation. Therefore, more granular data of potentially reversible ACLF are not available and should be studied in future research. Moreover, plasma cytokine levels were measured exclusively at the time of inclusion. Heterogeneous distribution of ILs and high rates of undetectable ILs were previously reported. ${ }^{1718}$ To the best of our knowledge no data from patients under the same conditions are available, and since in clinical practice we cannot exclude patients the fact that we did not exclude patients from the IL analysis is a strength of this study. Another strength is the supporting data from an experimental cirrhosis model as well as an external cohort. Such translationally congruent data on the development of ACLF in compensated and recompensated patients are unique in the current literature. 


\section{CONCLUSION}

This study shows that detectable levels of IL- $1 \alpha$ and- $1 \beta$ in clinically stable patients with cirrhosis are associated with a higher risk of ACLF development. Whereas IL- $1 \alpha$ is an independent predictor of ACLF development in compensated patients, IL-1 $\beta$ is a risk factor in recompensated patients, suggesting canonical and non-canonical inflammasome activation in different states of human and experimental liver cirrhosis.

\section{Author affiliations}

${ }^{1}$ Internal Medicine I, University of Bonn, Bonn, Germany

${ }^{2}$ Department of Medicine, Hospital Pedro Hispano, Matosinhos, Portugal

${ }^{3}$ Gastrounit Medical Division, Hvidovre Hospital, Hvidovre, Denmark

${ }^{4}$ Department of Internal Medicine 1, University of Frankfurt, Frankfurt am Main, Germany

${ }^{5}$ Department of Clinical Physiology and Nuclear Medicine, Centre for Functional and Diagnostic Imaging and Research, Hvidovre Hospital, Hvidovre, Denmark

${ }^{6}$ Department of Pharmacology, University of Frankfurt, Frankfurt am Main, Germany ${ }^{7}$ European Foundation for the Study of Chronic Liver Failure, Barcelona, Catalunya, Spain

${ }^{8}$ Liver Unit, Hospital Clínic, Barcelona, Spain

${ }^{9}$ Department of Biochemistry/Molecular Genetics, Hospital Clinic/University of Barcelona, Barcelona, Spain

${ }^{10}$ Hepatology, Hopital Beaujon, Clichy, France

${ }^{11}$ Institute for Liver and Digestive Health, UCL, London, UK

${ }^{12}$ Department of Clinical Physiology, Hvidovre Hospital and Faculty of Health

Sciences, University of Copenhagen, Herlev, Denmark

Twitter Frank Erhard Uschner @UschnerFrank, Robert Schierwagen @R_ Schierwagen and Gautam Mehta @drgautammehta

Acknowledgements We thank Gudrun Hack for excellent technical assistance and Sabine Dentler for critical reading.

Contributors SofM, JG, FEU: acquisition of data, analysis and interpretation of data, drafting of the manuscript, statistical analysis. NK, JLM, RS, SK, CW, LS, CJ, JC, JA-Q, VA, RM, JF, FB, GM: critical revision of the manuscript regarding important intellectual content. LLG, SorM, MP, JT: study concept and design, acquisition of data, analysis and interpretation of data, drafting of the manuscript, critical revision of the manuscript regarding important intellectual content, funding recipient, administrative, technical and material support, study supervision.LLG,SorM,MP,JT:contributed equally as last author

Funding The authors were supported by grants from Deutsche Forschungsgemeinschaft (SFB TRR57 P18, CRC 1382 A09, SFB 815 A5, SFB 1177 C02), Ernst-und-Berta-Grimmke Foundation, the European Union's Horizon 2020 research and innovation programme's GALAXY study (no 668031), LIVERHOPE (no 731875). MICROB-PREDICT (no 825694) and the Cellex Foundation (PREDICT). The funders had no influence on study design, data collection and analysis, decision to publish, or preparation of the manuscript.

Competing interests None declared.

Patient and public involvement Patients and/or the public were not involved in the design, or conduct, or reporting, or dissemination plans of this research.

Patient consent for publication Not required.

Ethics approval The study was approved by the local ethics committee and Data Protection Agency (J-No 2008-41-2020 and HVH-2011-02).

Provenance and peer review Not commissioned; externally peer reviewed.

Data availability statement All data relevant to the study are included in the article, uploaded as supplementary information, or available upon contacting the corresponding author.

Open access This is an open access article distributed in accordance with the Creative Commons Attribution Non Commercial (CC BY-NC 4.0) license, which permits others to distribute, remix, adapt, build upon this work non-commercially, and license their derivative works on different terms, provided the original work is properly cited, appropriate credit is given, any changes made indicated, and the use is non-commercial. See: http://creativecommons.org/licenses/by-nc/4.0/.

\section{ORCID iDs}

Frank Erhard Uschner http://orcid.org/0000-0002-3760-2887

Nina Kimer http://orcid.org/0000-0002-4807-1575

Robert Schierwagen http://orcid.org/0000-0002-2195-3666

Joan Claria http://orcid.org/0000-0003-4333-7749

Gautam Mehta http://orcid.org/0000-0002-5696-359X

Michael Praktiknjo http://orcid.org/0000-0001-7033-9956

Jonel Trebicka http://orcid.org/0000-0002-7028-3881

\section{REFERENCES}

1 Moreau R, Jalan R, Gines P, et al. Acute-On-Chronic liver failure is a distinct syndrome that develops in patients with acute decompensation of cirrhosis. Gastroenterology 2013; 144:1426-37.

2 European Association for the Study of the Liver. Electronic address: easloffice@ easloffice.eu, European Association for the Study of the LiverVillanueva C. EASL clinical practice guidelines for the management of patients with decompensated cirrhosis. J Hepatol 2018;69:406-60.

3 Praktiknjo M, Clees C, Pigliacelli A, et al. Sarcopenia is associated with development of acute-on-chronic liver failure in decompensated liver cirrhosis receiving transjugular intrahepatic portosystemic shunt. Clin Trans/ Gastroenterol 2019;10:e00025.

4 Praktiknjo M, Book M, Luetkens J, et al. Fat-Free muscle mass in magnetic resonance imaging predicts acute-on-chronic liver failure and survival in decompensated cirrhosis. Hepatology 2018;67:1014-26.

5 Úbeda M, Muñoz L, Borrero M-J, et al. Critical role of the liver in the induction of systemic inflammation in rats with preascitic cirrhosis. Hepatology 2010;52:2086-95

6 Albillos A, Lario M, Álvarez-Mon M. Cirrhosis-associated immune dysfunction: distinctive features and clinical relevance. J Hepatol 2014;61:1385-96.

7 Medzhitov R. Origin and physiological roles of inflammation. Nature 2008;454:428-35

8 Bernardi M, Moreau R, Angeli P, et al. Mechanisms of decompensation and organ failure in cirrhosis: from peripheral arterial vasodilation to systemic inflammation hypothesis. J Hepatol 2015;63:1272-84.

9 Arroyo V, Moreau R, Jalan R, et al. Acute-On-Chronic liver failure: a new syndrome that will re-classify cirrhosis. J Hepatol 2015;62:S131-43.

10 Piano $S$, Tonon $M$, Vettore $E$, et al. Incidence, predictors and outcomes of acute-onchronic liver failure in outpatients with cirrhosis. J Hepatol 2017;67:1177-84.

11 Heneka MT, McManus RM, Latz E. Inflammasome signalling in brain function and neurodegenerative disease. Nat Rev Neurosci 2018;19:610-21.

12 Zhou Z, Xu M-J, Gao B. Hepatocytes: a key cell type for innate immunity. Cell Mol Immunol 2016;13:301-15.

13 Rosenbloom AJet al. Leukocyte activation in the peripheral blood of patients with cirrhosis of the liver and SIRS. JAMA 1995;274:58.

14 Grebe A, Hoss F, Latz E. Nlrp3 inflammasome and the IL-1 pathway in atherosclerosis. Circ Res 2018; 122:1722-40.

15 D'Amico G, Morabito A, D'Amico M, et al. Clinical states of cirrhosis and competing risks. J Hepatol 2018;68:563-76.

16 Jansen C, Möller P, Meyer C, et al. Increase in liver stiffness after transjugular intrahepatic portosystemic shunt is associated with inflammation and predicts mortality. Hepatology 2018;67:1472-84.

17 Clària J, Stauber RE, Coenraad MJ, et al. Systemic inflammation in decompensated cirrhosis: characterization and role in acute-on-chronic liver failure. Hepatology 2016:64:1249-64.

18 Solé C, Solà E, Morales-Ruiz M, et al. Characterization of inflammatory response in acute-on-chronic liver failure and relationship with prognosis. Sci Rep 2016;6:32341.

19 Alcaraz-Quiles J, Titos E, Casulleras M, et al. Polymorphisms in the IL-1 gene cluster influence systemic inflammation in patients at risk for acute-on-chronic liver failure. Hepatology 2017:65:202-16.

20 Trebicka J, Hennenberg M, Odenthal M, et al. Atorvastatin attenuates hepatic fibrosis in rats after bile duct ligation via decreased turnover of hepatic stellate cells. J Hepatol 2010:53:702-12.

21 Trebicka J, Hennenberg M, Laleman W, et al. Atorvastatin lowers portal pressure in cirrhotic rats by inhibition of RhoA/Rho-kinase and activation of endothelial nitric oxide synthase. Hepatology 2007;46:242-53.

22 Beyer M, Abdullah Z, Chemnitz JM, et al. Tumor-necrosis factor impairs CD4(+) T cell-mediated immunological control in chronic viral infection. Nat Immuno 2016;17:593-603.

23 Trebicka J, Amoros A, Pitarch C, et al. Addressing profiles of systemic inflammation across the different clinical phenotypes of acutely decompensated cirrhosis. Front Immunol 2019;10:00476.

24 Di Paolo NC, Shayakhmetov DM. Interleukin $1 \alpha$ and the inflammatory process. Nat Immunol 2016:17:906-13.

25 Rider $\mathrm{P}$, Carmi Y, Guttman 0, et al. IL-1 $\alpha$ and IL-1 $\beta$ recruit different myeloid cells and promote different stages of sterile inflammation. J Immunol Baltim Md 2011;1950:4835-43.

26 Garlanda C, Dinarello CA, Mantovani A. The interleukin-1 family: back to the future. Immunity 2013;39:1003-18.

27 Kurt-Jones EA, Beller DI, Mizel SB, et al. Identification of a membrane-associated interleukin 1 in macrophages. Proc Natl Acad Sci U S A 1985;82:1204-8.

28 Rider $\mathrm{P}$, Kaplanov I, Romzova M, et al. The transcription of the alarmin cytokine interleukin-1 alpha is controlled by hypoxia inducible factors 1 and 2 alpha in hypoxic cells. Front Immunol 2012:3:290.

29 Bersudsky M, Luski L, Fishman D, et al. Non-Redundant properties of IL-1 $\alpha$ and IL-1 $\beta$ during acute colon inflammation in mice. Gut 2014;63:598-609.

$30 \mathrm{Kim} B$, Lee $Y, \operatorname{Kim} E$, et al. The interleukin- $1 \alpha$ precursor is biologically active and is likely a key alarmin in the IL-1 family of cytokines. Front Immunol 2013:4:391. 
31 Malik A, Kanneganti T-D. Function and regulation of IL-1 $\alpha$ in inflammatory diseases and cancer. Immunol Rev 2018;281:124-37.

32 Chen C-J, Kono H, Golenbock D, et al. Identification of a key pathway required for the sterile inflammatory response triggered by dying cells. Nat Med 2007;13:851-6.

33 Wiggins KA, Parry AJ, Cassidy LD, et al. IL- $1 \alpha$ cleavage by inflammatory caspases of the noncanonical inflammasome controls the senescence-associated secretory phenotype. Aging Cell 2019;18:e12946.

34 Kelley N, Jeltema D, Duan Y, et al. The NLRP3 inflammasome: an overview of mechanisms of activation and regulation. Int J Mol Sci 2019;20:E3328.

35 Turco L, Garcia-Tsao G, Magnani I, et al. Cardiopulmonary hemodynamics and C-reactive protein as prognostic indicators in compensated and decompensated cirrhosis. J Hepatol 2018;68:949-58.

36 Swanson KV, Deng M, Ting JP-Y. The NLRP3 inflammasome: molecular activation and regulation to therapeutics. Nat Rev Immunol 2019;19:477-89.
37 Xing Y, Yao X, Li H, et al. Cutting edge: TRAF6 mediates TLR/IL-1R SignalingInduced nontranscriptional priming of the NLRP3 inflammasome. J Immunol 2017;199:1561-6.

38 Macdonald S, Andreola F, Bachtiger $\mathrm{P}$, et al. Cell death markers in patients with cirrhosis and acute decompensation. Hepatology 2018;67:989-1002.

39 Piccini A, Carta S, Tassi S, et al. ATP is released by monocytes stimulated with pathogen-sensing receptor ligands and induces IL-1beta and IL-18 secretion in an autocrine way. Proc Natl Acad Sci U S A 2008;105:8067-72.

40 Netea MG, Nold-Petry CA, Nold MF, et al. Differential requirement for the activation of the inflammasome for processing and release of IL-1beta in monocytes and macrophages. Blood 2009;113:2324-35.

41 Kamath PS, Wiesner RH, Malinchoc M, et al. A model to predict survival in patients with end-stage liver disease. Hepatology 2001;33:464-70.

42 Jalan R, Pavesi M, Saliba F, et al. The CLIF Consortium acute decompensation score (CLIF-C ads) for prognosis of hospitalised cirrhotic patients without acute-on-chronic liver failure. J Hepatol 2015;62:831-40. 